\title{
Intentionally Increased Flexion Angle of the Femoral Component in Mobile Bearing Unicompartmental Knee Arthroplasty
}

\author{
Kye-Youl Cho, $\mathrm{MD}^{1,2}$, Kang-Il Kim, $\mathrm{MD}^{2,3}$, Sang-Jun Song, $\mathrm{MD}^{3}$, and Kyu-Jin Kim, $\mathrm{MD}^{2}$ \\ ${ }^{1}$ Department of Medicine, Graduate School, Kyung Hee University, Seoul; ${ }^{2}$ Department of Orthopaedic Surgery, Center for Joint Diseases and Rheumatism, Kyung Hee \\ University Hospital at Gangdong, Seoul, ${ }^{3}$ Department of Orthopaedic Surgery, Kyung Hee University College of Medicine, Seoul, Korea
}

\begin{abstract}
Purpose: The purpose of this study was to determine the results of mobile bearing unicompartmental knee arthroplasty (UKA) with an intentionally increased flexion angle of the femoral component in patients requiring high flexion.

Materials and Methods: We investigated 45 knees treated by UKA. Clinically, we measured the range of motion (ROM) and the American Knee Society (AKS) score preoperatively and at final follow-up and investigated complications. Radiologically, we measured the flexion angle of the femoral component, the posterior slope angle of the tibial component, the femorotibial angle and mechanical axis of the limb postoperatively.

Results: The ROM was increased from $123^{\circ}$ preoperatively to $139^{\circ}$ at the final follow-up. The AKS knee and function scores increased from 59 and 68 , respectively, preoperatively to 94 and 96 , respectively, at the final follow-up. The flexion angle of the femoral component was $9.1^{\circ}$, and the posterior slope angle of the tibial component was $8.6^{\circ}$. There was one case of bearing dislocation in the largest femoral flexion angle case.

Conclusions: The results might reflect the positive effect of an increased flexion angle of the femoral component up to $10^{\circ}$ on ROM in mobile bearing UKA, which would contribute to better quality of life after UKA especially in populations requiring deep knee flexion.
\end{abstract}

Keywords: Knee, Arthroplasty, Unicompartmental, Flexion angle, Femoral component, Range of motion

\section{Introduction}

Better range of motion (ROM) following knee arthroplasty is an important factor with regard to better clinical outcomes ${ }^{1-3)}$. In Asian cultures, highly flexed knee positions are required more frequently than in other cultures because of cultural or religious reasons $^{1,3-5)}$. Theoretically, unicompartmental knee arthroplasty (UKA) has the benefit of increasing ROM compared to total knee

Received June 23, 2017; Revised August 21, 2017;

Accepted September 18, 2017

Correspondence to: Kang-Il Kim, MD

Department of Orthopaedic Surgery, Center for Joint Diseases and

Rheumatism, Kyung Hee University Hospital at Gangdong, 892

Dongnamro, Gangdong-gu, Seoul 05278, Korea

Tel: +82-2-440-6151, Fax: +82-2-440-6296

E-mail: drkim@khu.ac.kr

This is an Open Access article distributed under the terms of the Creative Commons Attribution Non-Commercial License (http://creativecommons.org/licenses/by-nc/4.0/) which permits unrestricted non-commercial use, distribution, and reproduction in any medium, provided the original work is properly cited. arthroplasty (TKA) ${ }^{6-8)}$; however, high flexion can also increase the rate of complications such as dislocation of the polyethylene bearing in mobile bearing systems ${ }^{4,5)}$. Currently, improved operative techniques and new implants have been developed to permit higher knee flexion and to minimize complications ${ }^{9-11)}$.

In general, increased flexion angle of femoral components may permit higher flexion ${ }^{3)}$. However, as the angle increases, the risk of dislocation can also increase due to subsequently increased flexion gap with abnormal gap balancing in mobile bearing systems ${ }^{4,12)}$. Given these facts, the Oxford group suggested a flexion angle of the femoral component between $5^{\circ}$ extension and $10^{\circ}$ flexion for mobile bearing $\mathrm{UKA}^{13)}$. Many articles regarding mobile bearing UKA have reported the mean flexion angle of the femoral component ranged between $0.8^{\circ}$ extension and $2.1^{\circ}$ flexion in their series ${ }^{13-16)}$. Those angles are close to neutral $0^{\circ}$ flexion and far from the $10^{\circ}$ flexion of acceptable limit suggested by them. Nonetheless, we could not find any clinical report presenting beyond $2.1^{\circ}$ of average femoral component flexion angle in mobile bearing UKAs. 
The purpose of this study was to determine the effects of mobile bearing UKA in patients requiring high flexion knees, receiving about $10^{\circ}$ of flexion angle of the femoral component which is the nearest numerical value as acceptable limit in flexion angle of the femoral component suggested by the Oxford group ${ }^{13)}$. We hypothesized that the intentionally increased angle of the femoral component close to $10^{\circ}$ of flexion would increase the postoperative ROM (Fig. 1) without increasing the rate of bearing dislocation or additional complications. To support the hypothesis, we compared our findings with those presented in the literatures on flexion angles of the femoral component in mobile bearing UKA.

\section{Materials and Methods}

We retrospectively investigated 43 patients ( 45 knees) treated by UKA using Oxford phase 3 (Biomet, Bridgend, UK) components. The mean follow-up period was 51 months (range, 23 to 75 months), except one patient due to death from lung cancer. There were 7 males and 35 females with a mean age of 61 years (range, 48 to 78 years). Written informed consent was obtained from all patients before this institutionally approved study was initiated. The preoperative diagnosis was medial unicompartmental osteoarthritis of the knee in all cases. The operation was performed by a senior author in all cases.

We assessed the preoperative magnetic resonance imaging scans in all patients to verify the status of cruciate ligaments, menisci and degenerative changes in the cartilaginous lesions. All patients had varus deformities and flexion contractures less than $15^{\circ}$ with ROM greater than $100^{\circ}$ preoperatively ${ }^{6}$. Patients with asymptomatic degenerative changes of the patellofemoral joint were included ${ }^{6,8)}$. We excluded patients with anterior or posterior instability and those with grade 2 degenerative lesions in the lateral compartment according to the Kellgren and Lawrence classification $^{6,8)}$.

For the operation, about $8 \mathrm{~cm}$ longitudinal incision was made at about $1 \mathrm{~cm}$ medial to the proximal part of the patella. After opening the joint, without everting the patella, we removed all osteophytes and verified the status of intraarticular structures such as cartilage, anterior cruciate ligament and menisci. Next, we performed a medial tibial cut perpendicular to the tibial mechanical axis using a tibial saw guide aimed at about $7^{\circ}$ of the posterior tibial slope angle. We then performed an excision of the anterior part of the medial meniscus. Then we drilled a hole at $1 \mathrm{~cm}$ anterior to the anteromedial corner of the intercondylar notch, inserted an intramedullary (IM) rod and positioned a femoral drill guide based on the IM rod. From a sagittal view, the uppermost surface of the drill guide had been recommended parallel to the IM rod, however, we positioned the drill guide at about $10^{\circ}$ flexed to the IM rod using a goniometer in the lateral view. Then we cut the posterior side of the medial femoral condyle using a cutting guide based on the drill guide with about $10^{\circ}$ flexion. We measured the flexion and extension gap using a filler gauge and matched the gap by gradual milling of the distal femoral condyle. After checking the balanced flexion and extension gap with a trial implant inserted, we fixed the real tibial and femoral components with bone cement and inserted the mobile bearing polyethylene. All patients performed ankle-pumping exercises and active motion exercises on the day of operation as well as passive motion

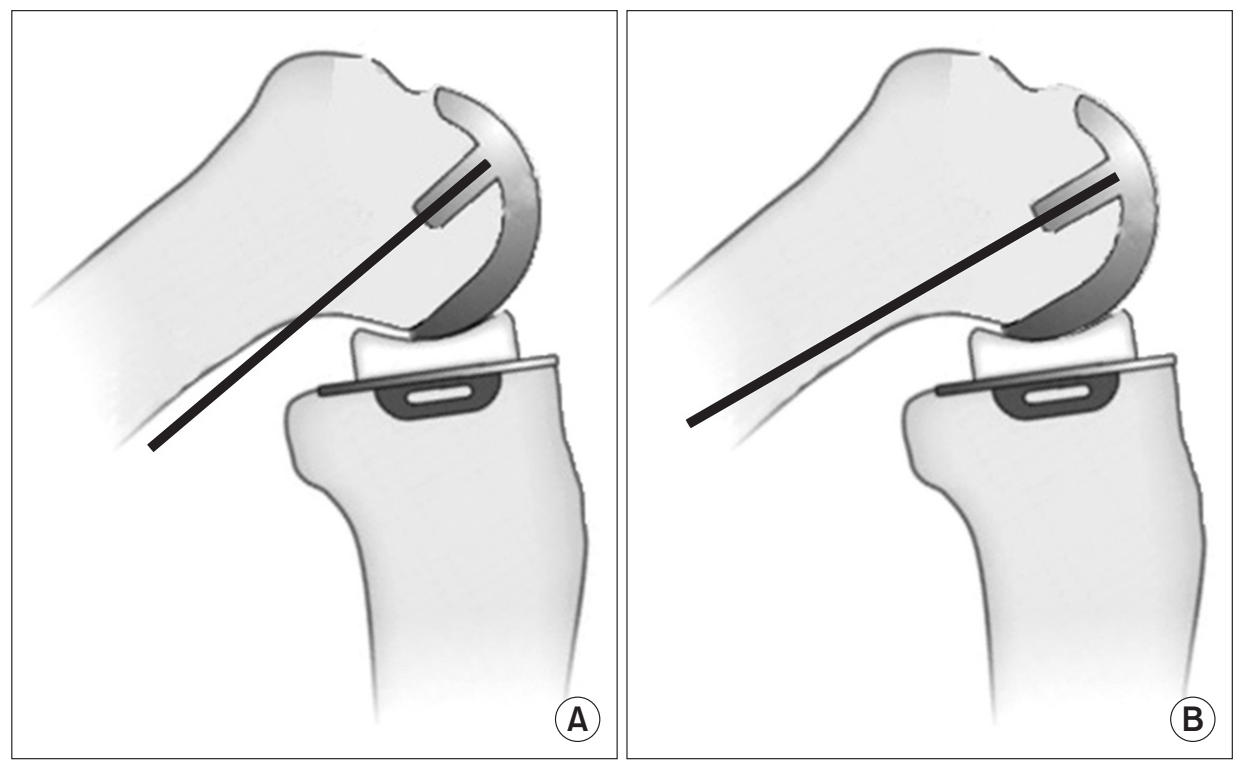

Fig. 1. Illustrations showing different sagittal positions of the femoral component. Flexed position of the femoral component can increase posterior contact surface and range of motion. (A) Neutral position. (B) Flexed position. 
exercises from postoperative day one. We educated patients on crutch ambulation and allowed pain-free distance ambulation. Outpatient follow-up was performed at 6 weeks, 3 months, 6 months, and 1 year postoperatively and then once every year. Radiologically, we measured the femorotibial angle pre- and postoperatively as well as the mechanical axis. Furthermore, we measured the flexion angle of the femoral component and posterior slope angle of the tibial component on postoperative radiographs. Clinically, we measured the American Knee Society (AKS) score and ROM preoperatively and at the latest follow-up and investigated complications including polyethylene bearing dislocation at the last follow-up.

\section{Results}

The mean preoperative femorotibial angle was $2.5^{\circ}$ valgus (range, $4.8^{\circ}$ varus to $8.1^{\circ}$ valgus), which was corrected to $6.0^{\circ}$ valgus (range, $0.2^{\circ}$ valgus to $12.8^{\circ}$ valgus) postoperatively, with the mean preoperative mechanical axis of $4.8^{\circ}$ varus (range, $12.0^{\circ}$ varus to $3.1^{\circ}$ valgus) corrected to $0.7^{\circ}$ varus (range, $6.7^{\circ}$ varus to $6.9^{\circ}$ valgus). The average flexion angle of the femoral component was $9.1^{\circ}$ (range, $5.0^{\circ}$ to $15.3^{\circ}$ ), and the average posterior slope angle of the tibial component was $8.6^{\circ}$ (range, $4.6^{\circ}$ to $10.0^{\circ}$ ).

The average AKS knee score increased from 59 (range, 52 to 70 ) preoperatively to 94 (range, 70 to 100) and the average AKS function score increased from 68 (range, 40 to 70 ) to 96 (range, 80 to 100 ) at the last follow-up. The average ROM was increased from $123^{\circ}$ to $139^{\circ}$ and the flexion contracture decreased from $4.7^{\circ}$ to $0^{\circ}$ at the last follow-up (Table 1).

There was one case of bearing dislocation. It developed at post- operative 6 weeks in a patient with $15.3^{\circ}$ of flexion of the femoral component which was the largest flexion angle among our series. Consequently, it was converted to TKA. There was no other additional postoperative complication such as infection or early implant loosening till the last follow-up.

\section{Discussion}

The principal finding of this study was that a better ROM was achieved with an intentionally increased flexion angle of the femoral component in mobile bearing UKA. To achieve satisfactory clinical outcomes with UKA, it is crucial to determine the proper position of components ${ }^{17-19)}$. Radiologically, the femoral component's varus/valgus angle or mediolateral distance in the coronal plane is important because of impingement or edge loading on the polyethylene bearing especially in fixed bearing $\mathrm{UKA}^{14,15}$. However, there has been little knowledge about the sagittal positioning of the femoral components, and much contention exists surrounding the normal ranges of femoral component flexion and extension angles ${ }^{13)}$.

In the current study, we hypothesized that the postoperative ROM would increase after UKA performed with the target femoral component angle of $10^{\circ}$ as suggested by the Oxford group as the acceptable high flexion angle of the femoral component ${ }^{13)}$. We conjectured that increasing the flexion angle of femoral components would facilitate better flexion of the knee joints through gradual but stable widening and lengthening of the articular surface in contact with the posterior surface of the femoral component and bearing during deep knee flexion (Fig. 1). Up to now, existing literatures have reported average flexion angle of the

Table 1. Comparison of Previous Studies about Component Angles, Range of Motion (ROM) and Dislocation

\begin{tabular}{|c|c|c|c|c|c|}
\hline \multirow{2}{*}{ Study } & \multirow{2}{*}{$\begin{array}{l}\text { Flexion angle of femoral } \\
\text { component }\left(\left(^{\circ}\right)\right.\end{array}$} & \multirow{2}{*}{ Posterior tibial slope $\left(^{\circ}\right)$} & \multicolumn{2}{|c|}{$\operatorname{ROM}\left(^{\circ}\right)$} & \multirow{2}{*}{$\begin{array}{c}\text { No. of dislocations } \\
(\%)\end{array}$} \\
\hline & & & Preoperative & Postoperative & \\
\hline Shakespeare et al. ${ }^{16)}$ & $-0.2(-10$ to 15$)$ & $5.7(-5$ to 10$)$ & N/A & N/A & N/A \\
\hline Vorlat et al. ${ }^{23)}$ & N/A & N/A & N/A & N/A & $4(2.6)$ \\
\hline Gulati et al. ${ }^{15)}$ & $-0.8(-9.9$ to 9.6$)$ & $5.1(0.8$ to 13.1$)$ & N/A & N/A & $0(0)$ \\
\hline Clarius et al. ${ }^{14)}$ & $2.1(-10$ to 19$)$ & $6.1(0$ to 14$)$ & 115 (80 to 150$)$ & $130(100$ to 155$)$ & $\mathrm{N} / \mathrm{A}$ \\
\hline Pandit et al. ${ }^{22)}$ & N/A & N/A & 117 (25 to 145$)$ & $130(85$ to 152$)$ & $6(0.6)$ \\
\hline Choy et al. ${ }^{4)}$ & N/A & N/A & $135(95$ to 150$)$ & $150(140$ to 165$)$ & $10(5.3)$ \\
\hline Kim et al. ${ }^{25)}$ & N/A & N/A & $129(120$ to 135$)$ & $133(127$ to 150$)$ & $3(2.4)$ \\
\hline Lim et al. ${ }^{5)}$ & N/A & N/A & $129(120$ to 135$)$ & $133(127$ to 150$)$ & $12(3)$ \\
\hline Kim et al. ${ }^{13)}$ & $1.2(-12$ to 20$)$ & N/A & N/A & N/A & N/A \\
\hline Current study & $9.1(5.0$ to 15.3$)$ & $8.6(4.6$ to 10.0$)$ & $123(90$ to 140$)$ & $139(125$ to 145$)$ & $1(2.3)$ \\
\hline
\end{tabular}

Values are presented as mean (range).

N/A: not available. 
femoral components between $0.8^{\circ}$ extension and $2.1^{\circ}$ flexion $^{13-16)}$. Therefore, we intended to evaluate the postoperative ROM of the knee with an intentionally increased flexion angle of the femoral component of about $10^{\circ}$ and compare with previous reports.

The postoperative ROM documented in previous Western articles using the Oxford phase 3 ranges from $130^{\circ}$ to $133^{\circ 8,14,20)}$. Among Asian studies, Lim et al. $^{5)}$ reported $133^{\circ}$ of postoperative ROM compared to $129^{\circ}$ of preoperative ROM, and Kim et $\mathrm{al}^{21)}$ reported $133.5^{\circ}$ as a mean postoperative ROM. The average postoperative $\mathrm{ROM}$ in the current study $\left(139^{\circ}\right)$ was in agreement with the results of these previous articles. However, there was no information on the flexion angle of the femoral component in those studies ${ }^{5,8,20,21)}$. Therefore, we could not compare with those studies in term of radiographic flexion angle of the femoral component.

There were few articles reporting the femoral component angle in the sagittal plane. To the best of our knowledge, only one article by Clarius et al. ${ }^{14)}$ reported a relationship between flexionextension angles of the femoral components and clinical scores in UKA using Oxford phase 3 implants. They inserted femoral components with an average $2.1^{\circ}$ of flexion and there was no difference in clinical scores between the properly implanted group and the outlier group according to the guidelines proposed by the Oxford group ${ }^{14)}$. In all the other reports with Oxford phase 3 , the radiographic mean flexion angle of the femoral components was considerably lower than that in the current study ${ }^{14-16)}$ (Table 1). Among these, comparison on the postoperative ROM was possible only with the study of Clarius et al. ${ }^{14)}$ : postoperative knee flexion was greater in our study compared to the study with a different flexion angle of the femoral component. This might indicate the positive effect of the increased flexion angle of the femoral component on postoperative ROM.

Although an increased flexion angle of the femoral component may allow a better ROM of the knee joint ${ }^{3)}$, bearing dislocation can occur due to the increased flexion gap resulting from gradual widening of the flexion gap in deep flexion ${ }^{4)}$. In the current study, the flexion angle of the components was aimed at about $10^{\circ}$, which is the maximum permissible angle according to the Oxford group recommendation ${ }^{13)}$, and there was no dislocation observed up to this angle. We experienced one case of bearing dislocation, but it was an exceptional case with $15.3^{\circ}$ of flexion of the femoral component; this was far beyond our target angle and was the largest flexion angle in our series. So, we carefully suggest that the risk of bearing dislocation can increase in case of overly greater flexion angle of the femoral component.

The incidence of bearing dislocation in mobile bearing UKA was $0.6 \%$ to $2.6 \%$ in recent studies ${ }^{22-25)}$. However, considering these results were all from the Western countries, it has limited applicability to Asian countries where kneeling and cross-legged positions are required much more frequently. Indeed, the studies in East Asia reported dislocation rates of $3 \%$ to $5.3 \%, 4,5)$, which might suggest the influence of different lifestyles on the rate of dislocation in different populations. On the other hand, Lim et al. ${ }^{5)}$ reported the non-anatomical bearing resulted in a higher rate of dislocation in the early period than the anatomical bearing (3.2\% vs. $2.8 \%)$. Similarly, Choy et al. ${ }^{4)}$ also suggested the impact of non-anatomical bearing on dislocation in their series. Therefore, we think the non-anatomic type of bearing could be considered as one of the causative factors of bearing dislocation.

The newly designed Microplasty (Zimmer Biomet, Bridgend, UK), the twin peg Oxford partial knee, adopted the extra peg, lengthened the posterior flange and the arc by $15^{\circ}$ and consequently increased the contact with a bearing at high knee flexion $^{11)}$. Although White et al. ${ }^{11)}$ reporting the new twin peg design with a $5.4^{\circ}$ of flexion angle of the femoral component did not demonstrate an increase in the actual ROM compared to the conventional one peg design, we anticipate an increased postoperative ROM in the twin peg design model with an intentionally increased flexion angle of about $10^{\circ}$ based on our results.

The limitations of our study include a relatively small number of cases (45 knees) without a comparison group; therefore, we could not compare with knees with a neutral flexion angle of the femoral components in a single surgeon series. On the risk of dislocation, other related factors such as the angle of posterior tibial slope or the tension of medial ligamentous structures were not investigated thoroughly. However, the degree and range of posterior tibial slope were similar among patients included in the current study and the operation technique was the same in all cases since it was a single surgeon's series. Last, the follow-up period was relatively short for arthroplasty, and therefore further investigation with a longer term follow-up would be required.

\section{Conclusions}

We think that the technique of intentionally increasing the femoral component flexion angle to about $10^{\circ}$ in mobile bearing UKA may produce a better ROM without increasing the incidence of bearing dislocation. This would contribute to better quality of life after UKA especially in the population demanding deep knee flexion. 


\section{Conflict of Interest}

No potential conflict of interest relevant to this article was reported.

\section{References}

1. Ueo T, Kihara Y, Ikeda N, Kawai J, Nakamura K, Hirokawa S. Deep flexion-oriented bisurface-type knee joint and its tibial rotation that attributes its high performance of flexion. J Arthroplasty. 2011;26:476-82.

2. Miner AL, Lingard EA, Wright EA, Sledge CB, Katz JN; Kinemax Outcomes Group. Knee range of motion after total knee arthroplasty: how important is this as an outcome measure? J Arthroplasty. 2003;18:286-94.

3. Kurosaka M, Yoshiya S, Mizuno K, Yamamoto T. Maximizing flexion after total knee arthroplasty: the need and the pitfalls. J Arthroplasty. 2002;17(4 Suppl 1):59-62.

4. Choy WS, Kim KJ, Lee SK, Yang DS, Lee NK. Mid-term results of oxford medial unicompartmental knee arthroplasty. Clin Orthop Surg. 2011;3:178-83.

5. Lim HC, Bae JH, Song SH, Kim SJ. Oxford phase 3 unicompartmental knee replacement in Korean patients. J Bone Joint Surg Br. 2012;94:1071-6.

6. Murray DW. Mobile bearing unicompartmental knee replacement. Orthopedics. 2005;28:985-7.

7. Newman J, Pydisetty RV, Ackroyd C. Unicompartmental or total knee replacement: the 15 -year results of a prospective randomised controlled trial. J Bone Joint Surg Br. 2009;91: 52-7.

8. Pandit H, Jenkins C, Barker K, Dodd CA, Murray DW. The Oxford medial unicompartmental knee replacement using a minimally-invasive approach. J Bone Joint Surg Br. 2006;88: 54-60.

9. Murray DW, Goodfellow JW, O’Connor JJ. The Oxford medial unicompartmental arthroplasty: a ten-year survival study. J Bone Joint Surg Br. 1998;80:983-9.

10. Price AJ, Webb J, Topf H, Dodd CA, Goodfellow JW, Murray DW; Oxford Hip and Knee Group. Rapid recovery after oxford unicompartmental arthroplasty through a short incision. J Arthroplasty. 2001;16:970-6.

11. White SH, Roberts S, Jones PW. The Twin Peg Oxford partial knee replacement: the first 100 cases. Knee. 2012;19:36-40.

12. Lewold S, Goodman S, Knutson K, Robertsson O, Lidgren L. Oxford meniscal bearing knee versus the Marmor knee in unicompartmental arthroplasty for arthrosis: a Swedish multicenter survival study. J Arthroplasty. 1995;10:722-31.

13. Kim JG, Kasat NS, Bae JH, Kim SJ, Oh SM, Lim HC. The radiological parameters correlated with the alignment of the femoral component after Oxford phase 3 unicompartmental knee replacement. J Bone Joint Surg Br. 2012;94:1499-505.

14. Clarius M, Hauck C, Seeger JB, Pritsch M, Merle C, Aldinger PR. Correlation of positioning and clinical results in Oxford UKA. Int Orthop. 2010;34:1145-51.

15. Gulati A, Chau R, Simpson DJ, Dodd CA, Gill HS, Murray DW. Influence of component alignment on outcome for unicompartmental knee replacement. Knee. 2009;16:196-9.

16. Shakespeare D, Ledger M, Kinzel V. Accuracy of implantation of components in the Oxford knee using the minimally invasive approach. Knee. 2005;12:405-9.

17. Kaya Bicer E, Servien E, Lustig S, Demey G, Ait Si Selmi T, Neyret P. Sagittal flexion angle of the femoral component in unicompartmental knee arthroplasty: is it same for both medial and lateral UKAs? Knee Surg Sports Traumatol Arthrosc. 2010;18:928-33.

18. Bert JM. 10-year survivorship of metal-backed, unicompartmental arthroplasty. J Arthroplasty. 1998;13:901-5.

19. Cartier P, Sanouiller JL, Grelsamer RP. Unicompartmental knee arthroplasty surgery. 10-year minimum follow-up period. J Arthroplasty. 1996;11:782-8.

20. Rees JL, Price AJ, Beard DJ, Dodd CA, Murray DW. Minimally invasive Oxford unicompartmental knee arthroplasty: functional results at 1 year and the effect of surgical inexperience. Knee. 2004;11:363-7.

21. Kim KT, Lee S, Park HS, Cho KH, Kim KS. A prospective analysis of Oxford phase 3 unicompartmental knee arthroplasty. Orthopedics. 2007;30(5 Suppl):15-8.

22. Pandit H, Jenkins C, Gill HS, Barker K, Dodd CA, Murray DW. Minimally invasive Oxford phase 3 unicompartmental knee replacement: results of 1000 cases. J Bone Joint Surg Br. 2011;93:198-204.

23. Vorlat P, Putzeys G, Cottenie D, Van Isacker T, Pouliart N, Handelberg F, Casteleyn PP, Gheysen F, Verdonk R. The Oxford unicompartmental knee prosthesis: an independent 10year survival analysis. Knee Surg Sports Traumatol Arthrosc. 2006;14:40-5.

24. Price AJ, Waite JC, Svard U. Long-term clinical results of the medial Oxford unicompartmental knee arthroplasty. Clin Orthop Relat Res. 2005;(435):171-80.

25. Kim SJ, Bae JH, Lim HC. Factors affecting the postoperative limb alignment and clinical outcome after Oxford unicompartmental knee arthroplasty. J Arthroplasty. 2012;27:1210-5. 